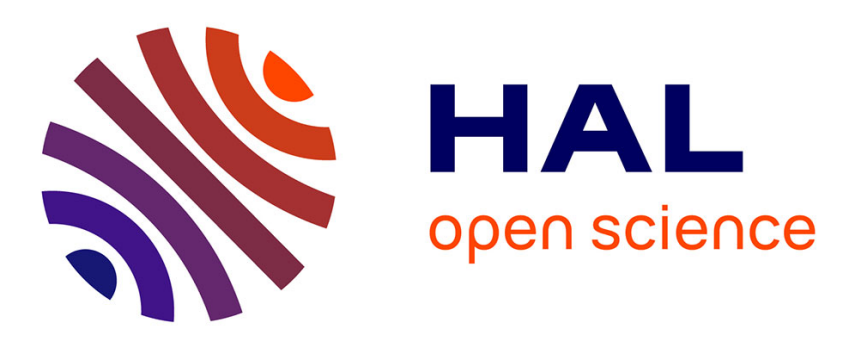

\title{
Design Practices and the SAwD Tool: Towards the Opendesign Concept
}

José Silva, Roberto Pereira, Elaine Hayashi, M. Baranauskas

\section{To cite this version:}

José Silva, Roberto Pereira, Elaine Hayashi, M. Baranauskas. Design Practices and the SAwD Tool: Towards the Opendesign Concept. 18th International Conference on Informatics and Semiotics in Organisations (ICISO), Jul 2018, Reading, United Kingdom. pp.208-217, 10.1007/978-3-319-945415_21. hal-01920715

\section{HAL Id: hal-01920715 https://hal.inria.fr/hal-01920715}

Submitted on 13 Nov 2018

HAL is a multi-disciplinary open access archive for the deposit and dissemination of scientific research documents, whether they are published or not. The documents may come from teaching and research institutions in France or abroad, or from public or private research centers.
L'archive ouverte pluridisciplinaire HAL, est destinée au dépôt et à la diffusion de documents scientifiques de niveau recherche, publiés ou non, émanant des établissements d'enseignement et de recherche français ou étrangers, des laboratoires publics ou privés. 


\title{
Design Practices and the Sawd Tool: Towards the Opendesign Concept
}

\author{
José Valderlei da Silva ${ }^{1}$, Roberto Pereira ${ }^{2}$, Elaine C. S. Hayashi ${ }^{1}$, \\ M. Cecília C. Baranauskas ${ }^{1}$ \\ ${ }^{1}$ Institute of Computing, University of Campinas (UNICAMP), Campinas, SP, Brazil \\ ${ }^{2}$ Federal University of Paraná (UFPR), Curitiba, PR, Brazil \\ 1 \{cecilia, vander.silva, hayashi\}@ic.unicamp.br; \\ 2rpereira@inf.ufpr.br
}

\begin{abstract}
The main idea behind the Open Source model of software development is to promote open collaboration in a decentralized process of coding. Varied endeavors have taken place in order to encourage, support and maintain the Open Source initiative, always focusing on the programming part of software development. Little to none has been said regarding decentralized, open collaboration in the system design process as a whole. Aiming at understanding what an OpenDesign initiative for information systems design would be, this article further explores the SAwD tool in a case study that considers the context of designing IoT solutions for airports. Organisational Semiotics and the Socially Aware Design constitute the theoretical basis of this work.
\end{abstract}

Keywords: Organisational Semiotics $•$ Socially Aware Design $\cdot$ Human-Computer Interaction • IoT

\section{Introduction}

The development of information systems pushed by its technological aspects is unfortunately a rather common practice. As the demand for computational systems grows, it becomes clearer that hardware and software may cause varied problems if developers skip phases. Early design phases as problem clarification and identification of interested parties can be vital for the success of a system. The lack of awareness in such phases of system development may imply profound social consequences, affecting society in many levels (economic, ethical, political, etc.). These problems can be avoided by not neglecting the social context of design.

The creation of systems that make sense to the target community of stakeholders demands the systemic view of socio technical approaches. These are approaches that recognize that the technical solution is an embedded part of a much more complex social system, from which behavior patterns, beliefs and values may rise.

Usually, software and hardware developers focus their work only on the technical aspects of development, often because they are not aware of design techniques that support this required broader view. Other developers do know about design techniques, 
but they lack specific knowledge or they cannot get hold of informants that can contribute by adding to the creation of a bigger picture. When it comes to the variety of participants exploring and discussing the social context of a technical solution, the more the merrier. This process, however, must be well organized and documented following methods that can support collaborative work.

Open collaboration is supported in the software development context of the Open Source communities. In this process, developers create their products by developing, using and/or modifying freely shared source codes. This decentralized process of software development offers benefits as optimized and enhanced quality, since many different improvements can be added to the original code, suggested by many different points of view.

While open source is a common practice in the development of system's code, it is much less common to find such practices in the system design process. This concept is being developed by the OpenDesign Project [2]. As part of the OpenDesign Project, this article explores earlier phases of design process as a preliminary work towards the characterization of the 'Open Design' concept. As part of this investigation, we have conducted two workshops in order to idealize IoT solutions for airports. The first workshop took place during the ICISO'16 [1] conference, which was held in University of Campinas, in the city of Campinas, in São Paulo, Brazil, in 2016. The second workshop was conducted as a masterclass during the IHC'17 symposium [6], in Joinville - Santa Catarina, Brazil, in 2017. Both workshops had the Socially Aware Design [3] (more on Section 2) as theoretical and methodological basis. Both workshops had the same goal: identify problems faced by people at airports with the objective of proposing conceptual solutions for an IoT system. The first workshop was focused mainly on problem clarification and we observed how artefacts could be used to promote discussions. The second workshop started with the same discussion on problem understanding and it went further, resulting in concepts for technological solutions.

Both workshops were conducted with participants working together in person; only afterwards were the ideas transcribed to the online tool $\mathrm{SAwD}^{1}$ (the Socially Aware Design [13]). SAwD is a CASE tool ${ }^{2}$ that supports cooperative work in early design activities. More specifically, SAwD supports the problem clarification and the proposal of computational solutions based on activities from Organisational Semiotics and Participatory Design.

These workshops and SAwD served as basis for a preliminary discussion that, along and throughout the 2 years of the OpenDesign Project, shall inform and direct the following questions: What characteristics compose the OpenDesign concept? and How can platforms support the OpenDesign to be developed?

The airport setting presents an interesting case context, especially when considering socio-technical approaches for design processes. An airport gathers people from different cultures who often do not share a common language for communication, but share interests (e.g. traveling) and difficulties (e.g. lost items).

\footnotetext{
${ }^{1}$ www.nied.unicamp.br/dsc

${ }^{2}$ Computer-aided software engineering (CASE) tool is a tool used in the design and/or development of applications.
} 
This article is organized as follows: Section 2 presents our theoretical, methodological and technical references; Section 3 describes the workshops; Section 4 informs the results from the workshops; Section 5 discusses the findings and Section 6 concludes.

\section{Organisational Semiotics, Socially Aware Computing and the SAwD tool}

The preliminary practices to explore methodologies and tools that support OpenDesign consider that Organizational Semiotics [14] and the Socially Aware Design [3] should be the basis for the evolution of the Open Design concept. Some of the artifacts already produced based on these theories are collaborative and participatory, involving stakeholders as co-designers. This section describes the theoretical and methodological background that guided our practices. They are the same ones that will be used as fundamental material for OpenDesing concepts and practices.

Organisational Semiotics is a branch of semiotics that "is based on the fundamental observation that all organised behavior is affected by people's communication and interpretation of signs, both individually and in groups" p. 24 [9]. Organisational Semiotics is positioned under the philosophical stance of the radical subjectivism [14][9]. In this paradigm reality is understood as a subjective and social product, constructed by agents' behaviors, which implies that "there is no knowledge without a knower" and "there is no knowing without action". Subjectivity and responsibility play important roles in this stance [8].

Designers are responsible for the impacts that a technological artefact brings to the community of users even when they are not aware of the reach of the consequences derived from its use. For that reason, it is vital to consider wider, holistic views in the design process. The Socially Aware Computing [3] calls for methods and artefacts to support the design that is aware of the social reaches of a technological solution. Not only technical aspects should be considered in the design process, but also informal and formal ones. Informal, formal and technical are the layers of the Semiotic Onion [15], one of the artefacts from Organisational Semiotics used in the Socially Aware Computing. A computational system (technical information system) is part of the formal part of an organisation, which in turn, is part of the whole organisation, including the informal part of it. The Semiotic Onion offers a structure that helps making explicit the system of beliefs, intentions, values and culture of the organisation, which are often neglected.

Other artefacts and techniques that can be used in initial stages of a design process are described in PAM - Problem Articulation Method [15][8]. PAM is part of MEASURE - Method for Eliciting Analysing and Specifying Users Requirements [8][15]. Among the techniques that compose PAM are the 'Stakeholder identification Diagram' and the 'Valuation framing'. As the name indicates, Stakeholders Identification Diagram (SID) helps in the process of identifying interested and relevant parties. The Valuation framing helps to reveal stakeholders' perspectives on ten different aspects of culture [9]. Similarly, to the Valuation framing, the Evaluation Framing [4] (EF) also puts into evidence stakeholders' perspectives, but in this technique problems 
are anticipated and ideas for solutions are generated. Another artifact is the Semiotic framework or the Semiotic Ladder (SL). SL provides a tool to help seeing information (signs) from different perspectives.

SID, EF and SL have been applied within Semio-participatory practices [3] in varied contexts in order to clarify the problem amongst stakeholders. SAwD was developed [13] in the endeavor of allowing remote participation of interested parties in an open and collaborative environment. For that, the artefacts were transferred and adapted to a digital format in an online usage. With SAwD participants are able to contribute in their own time and schedule, without the need to travel distances for physical encounters.

\section{The Evolution of the Airport Scenario: A Case Study}

As mentioned before, two workshops were held and in both of them, the scenario of an airport was used as the motif for the creation of an IoT system. Airports host an entire world in itself. They are equipped with many signals, installations and facilities to support arrivals and departures of people and objects from domestic and international flights. Airports must offer the entire infrastructure to accommodate passengers and their families and belongings, aircrafts, air companies' staff and other employees from the airport, etc. In order to provide a comfortable experience for passengers, airports offer terminals with a wide range of services and facilities as restrooms, stores, playgrounds, VIP lounges, emergency services, places for worship, museums, restaurants, coffee places, etc. International airports have to control, in addition, immigration, customs and passport control. People from all over the world can be found in airports. This means that, in a same physical environment, there are people from different cultures, who follow varied values and social rules. The differences go on: varied languages (spoken and signed), age, gender, sexual orientation, height and body size, travel experience, disabilities, abilities, etc. These people may interact among each other, with airport staff, and also they may interact with objects, as conveyor belts or baggage carousels, information panels and displays, gates, escalators, elevators, etc. These interactions allow passengers to reach common goals, for example, they may claim their luggage, find boarding gates, restrooms, etc. In the same way as people interact with 'things' in the airport, also airport things could interact with passengers and staff. Things could also interact with other things, being proactive in helping themselves, other people and employees, automatizing services like transportation of people and luggage, cleaning, security, etc.

The first workshop, proposed as a half-day activity, explored the use of SID and EF. Both artefacts were used with the objective of promoting discussions for problem understanding. In regards to $\mathrm{EF}$, participants were asked to converge the discussions to rather specific problems and their corresponding solutions and ideas. Problems and solutions should concern each stakeholder previously identified with SID.

The second workshop was a whole-day activity and had different participants. Moreover, in addition to SID and EF, other artifacts were used: SL and other participatory design techniques. The result was the proposal of concepts for IoT solutions for the airport scenario. For both workshops, the artifacts were printed as posters into which 
participants could place post-it's filled with their ideas and contributions. The two workshops are described as follows: Section 3.1 presents the first workshop, and Section 3.2, the second. The results are presented in section 4.

\subsection{Problem Clarification}

Twenty participants took part in the first workshop. Participants were from different cultures and countries (Brazil, China, Chile and others). The activity started with a discussion about the Socially Aware Design followed by an explanation on the design problem: the airport scenario. First participants brainstormed about the feelings (both positive and negative ones) related to trips,considering airports as the setting. This first moment helped directing the group towards a common problem. Once the scope was clear, participants were asked to consider the 'things' around an airport and the 'things' people carry on their trips. This should lead the discussions towards the definition of solutions for internet of things that could help passengers and other people related to the airport scenario.

Participants were invited to explore the IoT concept as a network in which objects exchange messages. It was highlighted that the things related to the airport scenario should provide support for people involved or affected during the actions of travelling. Therefore, the IoT should be thought in a more analytical manner: as the Internet of Human Things (IoHT). Most things are objects created by people to satisfy human needs. Some of these things are composed by other systems, from which people want information (e.g., information about the weather). When designing an IoT system, the concern is to embed technology in objects in order to exchange information and investigate events that occur in that environment. For this reason, the Human should be the center of attention when creating an IoT environment. In this sense, Internet of Things (IoT) becomes an Internet of Human Things (IoHT).

\subsection{From Problem Clarification to Concept Proposal}

In the second workshop twenty-four participants joined the discussions. They were all from Brazil, but from different regions. Like in the first workshop, the group was organized in two subgroups. Each group had their own set of posters. The difference from the first workshop is that this time discussions did not end at problem clarification, as the second workshop lasted all day. The groups went further, brainstorming concept ideas and preliminary interface elements for the envisioned system. At the end, each group presented their solutions regarding the IoT systems for the airport scenario.

After filling SID and EF, participatory design techniques were used to elicit ideas for the solution to be proposed. The techniques were: BrainWriting [5] and BrainDraw [10]. In the BrainWriting technique, a group sits in a circle and each member of the group starts writing their idea. A few seconds later, an alarm rings to inform that they should stop writing and pass the paper to the person next to them. This person will then read the content of the paper and either agree or disagree with it and then continue the idea by adding their own contribution until the alarm rings again. This goes on around the circle until the person receives back the paper he had started. The BrainDraw works 
in a similar way, but this time instead of writing, participants are supposed to draw user interface elements of the prospective system. The results from BrainWriting (main concepts) provide ideas for Brain Draw. Each group also had a flipchart in which they wrote notes on their ideas or used it to explain their thoughts. At the end of the activities, each group presented their results to the other.

\section{$4 \quad$ Results}

The first workshop provided important basis for the conduction of the second one. Having the same objective and scenario, the two workshops explored the use of varied artifacts from OS and participatory design practices. The first workshop explored SID and EF; while the second, besides SID and EF, also explored SL as well as concept ideas for applications. SID, EF and SL from both workshops were latter transcribed into the SAwD tool. The complete transcriptions are available in [7].

The first workshop resulted in the identification of stakeholders and related problems and solutions. One of the SIDs shows a general concern with safety: smoke detectors, earthquake alarms and security cameras were some of the ideas for the source information layer of SID. The other SID layers from the first workshop had many contributions directed to pets: pet's cage and accessories (contribution layer), health insurance for pets (source layer) and animal welfare department (community) are some of the results. Fig. 1 illustrates a moment from the first workshop.

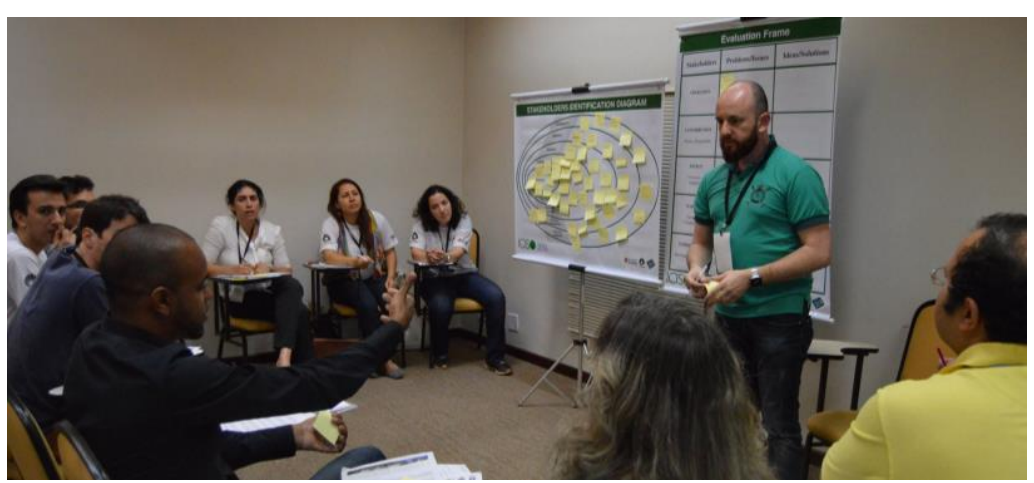

Fig. 1. Group discussion during the first workshop: SID (left) and EF (right).

The second workshop also resulted in the identification of stakeholders and possible problems and solutions. Moreover, it went further and continued with the discussion and proposition of concept ideas for IoT solutions. One of the solutions was based on the situation of a person traveling for the first time. The other, explored the problem of finding a connection at an unknown airport. For the first situation, participants considered how insecure and apprehensive passengers would be and idealized that this person would benefit from help for completing varied activities in the airport, like confirming flight schedule, checking in, dropping luggage and other boarding procedures. The proposal included a mobile application that would identify the person as he or she arrives 
at the airport. The group did not clearly define how the identification process would technically occur; options like retina scan, mobile phone and cards were discussed. $\mathrm{He}$ or she would then be guided to the places needed, which would be previously marked around the place. The application would use resources already available at airports and it should promote a stress-free travel experience for first-time passengers.

For the second situation, the proposal consisted in a personal assistant that should guide passengers during flight connections. Other orientations would also be provided: disaster procedures, indoor map, gate finding, time schedules, time organization, transfer to nearby airports, food services, etc. Such assistant would be composed of: mobile application, RFID tags, billboards, avatars and sensors (e.g. placed in plane's chairs). Interactions could take place via audio, text, images, sensors, signals, animations and/or vibrations. The application could be tailored for each user with multiple language resources.

\section{Discussion Towards Open Design}

Designers should be aware of the impacts that the insertion of a technological solution might bring about to a community of users [3]. With that in mind, when considering the expansion of the Open Source concept to Design processes, we argue that it is necessary that tools and platforms be developed in order to support the entire cycle, including early phases of the design process.

In order to start the investigation on the Open Design concept, we have used the SAwD tool to simulate the workshop activities in an online manner, including online versions of artifacts from Organizational Semiotics. For this exercise, the scenario of an airport was chosen. As mentioned before, airports present many interesting opportunities. It houses people with varied languages, cultures and values in a same place, bringing them close as they share similar objectives. Designing for such varied audience can be challenging, especially when accounting for a Socially Aware Design.

By observing the conduction of the two workshops that took place without digital technology, we were able to better understand the main characteristics that a platform or tool should have in order to make an online and collaborative Open Design Project a feasible experience. The transcription of the results from the artifacts from the workshops (EF, SID and Semiotic Framning) into the SAwD tool served as basis for a preliminary investigation and evaluation of SAwD as a potential platform for this purpose.

Concerning SID, the comparison between the poster filled during in-person activity and the SAwD tool filled afterwards (Fig. 2) indicates that the activity could be conducted online with the support of the tool. The SAwD supported the same input type in a collaborative way. During the workshop, each participant wrote their contribution in a post-it and stuck it on the SID poster. Some of them discussed the ideas before writing the post-it. In a similar way, participants would be able to fill SID with their contributions in SAwD. The online tool also supports discussions via the chat feature with one important advantage: the online chat can be retrieved later if needed. Even if one records the discussions in in-person activities, retrieving specific information would be easier in SAwD. 


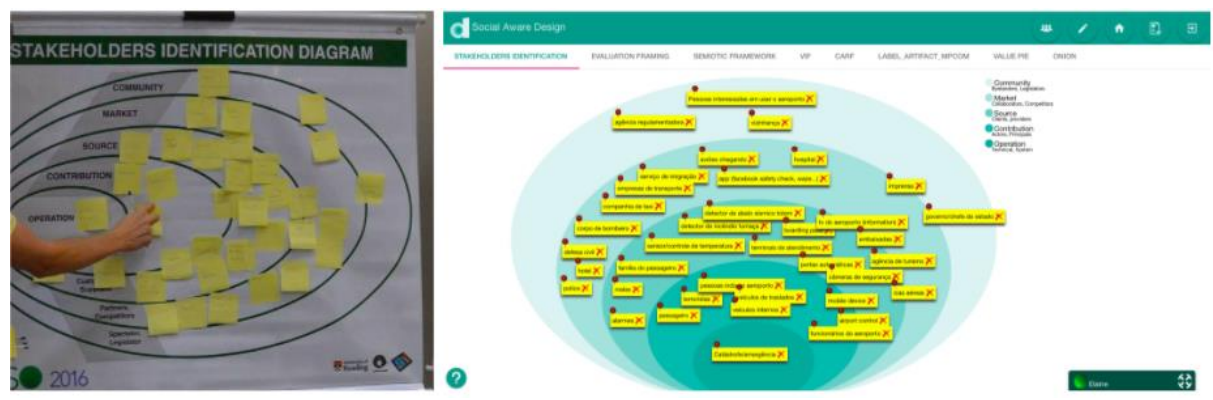

Fig. 2. From left to right: Poster of Stakeholders Identification Diagram being filled during the first workshop; the same artifact on the SAwD tool.

Differences were only found when handling the EF. The main difference observed was that the SAwD tool supports the interaction exactly as the artifact was intended for: it directly relates stakeholders to possible problems and ideas for solutions. When using the EF in person with post-it's, participants were able to insert problems at random without relating them to stakeholders. This is not possible in the present version of the SAwD tool. This difference between the original physical artefact and its online adaptation has positive and negative consequences. While SAwD does not reflect the interaction that happens in person, it forces the specified use of the artifact, making the activity more productive in terms of both effectiveness and efficiency.

Another important advantage of the SAwD tool is that it is easier to manipulate for recording and later analysis. When the workshops were over, the posters had to be rolled up and carried with care so that the post-it's would not be lost during transportation. Moreover, in order to read the results, one would need to open the entire poster and find a place to hang it. When dealing with an increased number of workshops, storing all the resulting posters becomes a troublesome task. Also the costs are higher in the in-person activity as it is necessary to have the posters with the artifacts printed; buy post-it's and pens; plus the additional costs of a physical installation (rent, facilities like restrooms and cleaning, etc.). This characteristic results in the main contribution of the SAwD tool in its value for an OpenDesign project. Since the artifacts in SAwD are easy to store and use, they are therefore easy to share. Other projects that are intended for similar scenarios can use the same results from a previous activity. The filled artifacts may serve as a starting point for different design projects. They may be used as they are or they may inspire other creations. Just like in Open Source projects, the SAwD tool will make it possible to leave the contributions for the Open Design community to use them as pleased or needed.

By analyzing the results from both workshops, which had the same central theme (airports), we can observe that some results were the same in both workshops. For example, as SID results both had passenger, attendant, etc.; and as EF results, time for check in and for landing, concerns with luggage, etc. In this sense, we can hypothesize that, if participants from the second workshop had had the chance to access the artifacts that resulted from the first workshop, they could have benefited from that knowledge 
and could have started with a preliminary understanding from the point of view of a different group. This would be possible with the SAwD tool.

From all of these advantages that were mentioned, in summary, one may affirm that the adaptations that were made so far to use the artifacts from Organizational Semiotics and Socially Aware Design as online tools were successful. The SAwD tool, as it is today, already provides support for all of the activities that usually takes place in faceto-face encounters. Moreover, it supports the concept of Open Design, as it allows sharing, coping, and editing the already existent design processes. However, more investigation is needed in order to enhance the SAwD tool even further. Tracking the decisions and changes that are made during the discussions could be a differential for the tool, as any contribution or change could be recovered. In addition, one of the challenges that is part of our future work is to include the role of a mediator. As it occurs in the face-to-face workshops, participants can profit from a mediator to inform and guide the discussions.

Only SF and EF were mentioned in this article; however, SAwD also supports dynamic online collaborative work with the Semiotic Onion [14][9][8] and Semiotic Ladder [14][8][9]; VIF [12]; CARF [12], Value Pie [11] and others.

\section{Conclusion}

The OpenDesing philosophy presupposes an open production in which what is produced belongs to everyone and not only to the creators of it. This means that a design solution can be extended or reapplied by others, whenever proponents agree to create an OpenDesign. The shared solution assumes that anyone can join or leave the team at any time. Each person can instantiate a design solution or part of it for a reuse of what has already been produced.

This article described two workshops that took place as in-person activities at a conference in 2016 and a symposium in 2017, to investigate the use of Organizational Semiotics and the Socially Aware Design artifacts to support ideas of Open Design. The analysis of the results and the comparison of the same activities using the online and collaborative tool SAwD indicate that SAwD has the potential of supporting the OpenDesign concept. This preliminary investigation has also indicated possibilities for the enhancement of the SAwD tool. Next steps include further development and evaluation of SAwD, as well as deeper investigation on the OpenDesign concept.

Acknowledgments. We would like to thank our colleagues from InterHad for insightful discussions. We also thank the financial support of CNPq (306272/2017-2); CAPES (1644511); and process grants \# (\#2015/24300-9) and (\#2015/16528-0) from São Paulo Research Foundation (FAPESP). 


\section{References}

1. Baranauskas, M. C. C., Liu, K., Sun, L., Neris, V. P. A., Bonacin, R., Nakata, K. (Eds.). (2016). Socially Aware Organisations and Technologies. Impact and Challenges: 17th IFIP WG 8.1 International Conference on Informatics and Semiotics in Organisations.

2. Baranauskas, M. C. C. (2015) OpenDesign: técnicas e artefatos para o design socialmente consciente de sistemas computacionais. Propostas FAPESP / MCTI / MC - 2015 Pesquisa Estratégica sobre a Internet 2015, \#2015/24300-9.

3. Baranauskas, M. C. C. (2014) Social awareness in HCI. Interactions, 21(4), 66-69.

4. Bonacin, R., Baranauskas, M.C.C., Santos, T.M. (2007) A Semiotic Approach for Flexible e-Government Service Oriented Systems. 9th ICEIS 2007. v. ISAS, 381-386.

5. Boy, G. A. (1997) The Group Elicitation Method for Participatory Design and Usability Testing. Interactions, march/april, 27-33.

6. Gasparini, I., Piccolo, L. S. G. (2017) IHC 2017 Emoção e Movimento - Proceedings of the 16th Brazilian Symposium on Human Factors in Computing Systems. To be published.

7. Hayashi, E. C. S.; Silva, J. V.; Pereira, R.; Baranauskas, M. C. C. (2018) Socially Aware Design in action - Practices and results. Technical Report, IC-/to be published.

8. Liu, K. (2000) Semiotics in Information Systems and Engineering. Cambridge University Press, Cambridge.

9. Liu, K.; Li, W. (2015) Organisational Semiotics for Business Informatics. Routledge, London.

10. Muller, M. (1997) Participatory Practices in the Software Lifecycle. Handbook of HumanComputer Interaction. Elsevier Science, 255-297.

11. Pereira, R., Baranauskas, M. C. C. (2014). Value pie: a culturally informed conceptual scheme for understanding values in design. International Conference on Human-Computer Interaction, pp. 122-133.

12. Pereira, R.; Buchdid, S. B.; Baranauskas, M. C. C. (2012). Values and cultural aspects in design: artifacts for making them explicit in design activities. International Conference on Enterprise Information Systems, pp. 358-375.

13. Silva, J. V.; Pereira, R.; Buchdid, S. B.; Duarte, E. F.; Baranauskas, M. C. C. (2016) SAwD - Socially Aware Design: An Organizational Semiotics-Based CASE Tool to Support Early Design Activities. Proc. of International Conference on Informatics and Semiotics in Organisations, ICISO 2016, v. 477, p. 59-69.

14. Stamper, R. (1993) A Semiotic Theory of Information and Information Systems. Invited papers for the ICL/University of Newcastle Seminar on Information.

15. Stamper, R., Liu, K., Hafkamp, M., Ades, Y. (2000) Understanding the roles of signs and norms in organisations: a semiotic approach to information systems design. Journal of Behavior and Information Technology 19 (1), 15-27. 